\title{
Digit sets of integral self-affine tiles with prime determinant
}

by

\author{
JiAN-Lin Li (Xi'an)
}

\begin{abstract}
Let $M \in M_{n}(\mathbb{Z})$ be expanding such that $|\operatorname{det}(M)|=p$ is a prime and $p \mathbb{Z}^{n} \nsubseteq M^{2}\left(\mathbb{Z}^{n}\right)$. Let $D \subset \mathbb{Z}^{n}$ be a finite set with $|D|=|\operatorname{det}(M)|$. Suppose the attractor $T(M, D)$ of the iterated function system $\left\{\phi_{d}(x)=M^{-1}(x+d)\right\}_{d \in D}$ has positive Lebesgue measure. We prove that (i) if $D \nsubseteq M\left(\mathbb{Z}^{n}\right)$, then $D$ is a complete set of coset representatives of $\mathbb{Z}^{n} / M\left(\mathbb{Z}^{n}\right)$; (ii) if $D \subseteq M\left(\mathbb{Z}^{n}\right)$, then there exists a positive integer $\gamma$ such that $D=$ $M^{\gamma} D_{0}$, where $D_{0}$ is a complete set of coset representatives of $\mathbb{Z}^{n} / M\left(\mathbb{Z}^{n}\right)$. This improves the corresponding results of Kenyon, Lagarias and Wang. We then give several remarks and examples to illustrate some problems on digit sets.
\end{abstract}

1. Introduction. Let $M \in M_{n}(\mathbb{Z})$ be an expanding matrix, that is, all eigenvalues of the integer matrix $M$ have moduli $>1$. For any finite subset $D \subset \mathbb{Z}^{n}$ of cardinality $|D|=|\operatorname{det}(M)|$, there exists a unique nonempty compact set $T:=T(M, D)$ such that

$$
M T=\bigcup_{d \in D}(T+d)
$$

More precisely, $T$ is the attractor of the iterated function system $\left\{\phi_{d}(x)=\right.$ $\left.M^{-1}(x+d)\right\}_{d \in D}$ (see [3]). A more explicit expression of $T$ is given by

$$
T(M, D)=\left\{\sum_{j=1}^{\infty} M^{-j} d_{j}: d_{j} \in D\right\}=\sum_{j=1}^{\infty} M^{-j} D .
$$

We call the above $D$ a digit set, based on the view that (1.2) gives a multidimensional generalization of a radix expansion for the members of $T$. For most pairs $(M, D)$, the set $T(M, D)$ has Lebesgue measure $\mu(T)=0$. If $T(M, D)$ has positive Lebesgue measure, we call $T(M, D)$ an integral self-affine tile. Associated with the pair $(M, D)$ is the smallest $M$-invariant sublattice of $\mathbb{Z}^{n}$ containing the difference set $D-D$, which we denote by $\mathbb{Z}[M, D]$. If $0 \in D$ then

$$
\mathbb{Z}[M, D]=\mathbb{Z}\left[D, M(D), \ldots, M^{n-1}(D)\right] .
$$

2000 Mathematics Subject Classification: Primary 28A80, 52C22; Secondary 11A63. Key words and phrases: iterated function system, integral self-affine tile, digit set. 
This follows from the Hamilton-Cayley theorem stating that $M^{n} \in \mathbb{Z}\left[M^{0}\right.$, $\left.M^{1}, \ldots, M^{n-1}\right]$. We call a digit set $D$ primitive (with respect to $M$ ) if $\mathbb{Z}[M, D]=\mathbb{Z}^{n}$. If $D^{\prime}=D-v$ for a constant vector $v \in \mathbb{Z}^{n}$, then $\mathbb{Z}\left[M, D^{\prime}\right]$ $=\mathbb{Z}[M, D]$. Without loss of generality, we may assume that $0 \in D$ by a translation. It follows from (1.3) that the condition $\mathbb{Z}[M, D] \nsubseteq M\left(\mathbb{Z}^{n}\right)$ is equivalent to $D \nsubseteq M\left(\mathbb{Z}^{n}\right)$.

It is known that most of the measure and tiling questions can be reduced to the case of primitive tiles. More precisely, Lagarias and Wang provide the following useful fact (see [6], [7, Theorem 1.2]).

Proposition 1. If the columns of a matrix $B \in M_{n}(\mathbb{Z})$ form a basis of $\mathbb{Z}[M, D]$, that is, $\mathbb{Z}[M, D]=B\left(\mathbb{Z}^{n}\right)$, then there exists a matrix $\widetilde{M}:=$ $B^{-1} M B \in M_{n}(\mathbb{Z})$ and a digit set $\widetilde{D}:=B^{-1} D \subset \mathbb{Z}^{n}$ such that $\mathbb{Z}[\widetilde{M}, \widetilde{D}]=\mathbb{Z}^{n}$, $0 \in \widetilde{D}$, and $T(M, D)=B(T(\widetilde{M}, \widetilde{D}))$.

In Proposition 1, the expanding integer matrix $M$ is changed to a different integer matrix $\widetilde{M}$, which is similar to $M$ over $\mathbb{Q}$ but not necessarily over $\mathbb{Z}$. Note that $B^{-1} \in M_{n}(\mathbb{Z})$ if and only if $D$ is primitive. We follow the terminology of [6], and say that $D$ is a standard digit set (with respect to $M)$ if $\widetilde{D}$ is a complete set of coset representatives of $\mathbb{Z}^{n} / \widetilde{M}\left(\mathbb{Z}^{n}\right)$. In this case, $\mu(T(M, D))=|\operatorname{det}(B)| \mu(T(\widetilde{M}, \widetilde{D}))>0$ (see [1]). A nonstandard digit set $D$ is one where $\mathbb{Z}[M, D]=B\left(\mathbb{Z}^{n}\right), T(M, D)=B(T(\widetilde{M}, \widetilde{D}))$ and $\mu(T(\widetilde{M}, \widetilde{D}))>0$, but $\widetilde{D}$ is not a complete set of coset representatives of $\mathbb{Z}^{n} / \widetilde{M}\left(\mathbb{Z}^{n}\right)$.

For the digit sets of integral self-affine tiles with prime determinant, Kenyon [4] proved the following:

TheOREM I. Let $p$ be a prime and $D \subset \mathbb{Z}$ be a primitive digit set with $|D|=|p|$. Then $T(p, D)$ is an integral self-affine tile if and only if $D$ is a complete set of residues mod $p$.

This result has been generalized by Lagarias and Wang [6, Theorem 4.1], [11, Theorem 4.2] who showed that nonstandard digit sets do not exist for many $M$ such that $|\operatorname{det}(M)|=p$ is a prime. In fact, they state the following.

Theorem II ([6, Theorem 4.1]). Let $M \in M_{n}(\mathbb{Z})$ be expanding such that $|\operatorname{det}(M)|=p$ is a prime and $p \mathbb{Z}^{n} \nsubseteq M^{2}\left(\mathbb{Z}^{n}\right)$. If $D \subset \mathbb{Z}^{n}$ is a digit set with $|D|=p$, then $\mu(T(M, D))>0$ if and only if $D$ is a standard digit set.

Since $p \mathbb{Z}^{n} \nsubseteq M^{2}\left(\mathbb{Z}^{n}\right)$ is equivalent to $p B^{-1}\left(\mathbb{Z}^{n}\right) \nsubseteq \widetilde{M}^{2}\left(B^{-1}\left(\mathbb{Z}^{n}\right)\right)$ which is not of the form $p \mathbb{Z}^{n} \nsubseteq \widetilde{M}^{2}\left(\mathbb{Z}^{n}\right)$, we observe that there is a gap in the proof of Theorem II in [6]. The proof there essentially yields the following.

Theorem III ([11, Theorem 4.2]). Let $M \in M_{n}(\mathbb{Z})$ be expanding such that $|\operatorname{det}(M)|=p$ is a prime and $p \mathbb{Z}^{n} \nsubseteq M^{2}\left(\mathbb{Z}^{n}\right)$. Let $D \subset \mathbb{Z}^{n}$ with $|D|=$ 
$|\operatorname{det}(M)|$ be primitive. Then $\mu(T(M, D))>0$ if and only if $D$ is a complete set of coset representatives of $\mathbb{Z}^{n} / M\left(\mathbb{Z}^{n}\right)$.

Since the sufficiency of the theorems above was proved by Bandt [1] under a much weaker condition, and the condition $p \mathbb{Z}^{n} \nsubseteq M^{2}\left(\mathbb{Z}^{n}\right)$ is always satisfied in the one-dimensional case, one often concentrates on the necessity of the theorems in higher dimensions (see [6, p. 174]). Based on the previous research concerning digit sets of integral self-affine tiles with prime determinant, in the present paper we first give a more general result which extends the above Theorems I-III. In particular, we obtain a complete proof of Theorem II. We then give several remarks and examples to illustrate some problems on digit sets.

2. Theorem and its proof. Our main result concerning the digit set $D$ of the integral self-affine tile $T(M, D)$ with prime $\operatorname{determinant} \operatorname{det}(M)$ is contained in the following.

TheOREM. Let $M \in M_{n}(\mathbb{Z})$ be expanding such that $|\operatorname{det}(M)|=p$ is a prime and $p \mathbb{Z}^{n} \nsubseteq M^{2}\left(\mathbb{Z}^{n}\right)$. Let $D \subset \mathbb{Z}^{n}$ be a finite set with $|D|=|\operatorname{det}(M)|$. Suppose that $T(M, D)$ is an integral self-affine tile. Then the following two conclusions hold:

(i) If $\mathbb{Z}[M, D] \nsubseteq M\left(\mathbb{Z}^{n}\right)$, then $D$ is a complete set of coset representatives of $\mathbb{Z}^{n} / M\left(\mathbb{Z}^{n}\right)$.

(ii) If $\mathbb{Z}[M, D] \subseteq M\left(\mathbb{Z}^{n}\right)$, then there exists a positive integer $\gamma$ such that $D=M^{\gamma} D_{0}$, where $D_{0}$ is a complete set of coset representatives of $\mathbb{Z}^{n} / M\left(\mathbb{Z}^{n}\right)$.

Note that either of the above conclusions (i) and (ii) implies that $D$ is a standard digit set, hence we obtain Theorem II. On the other hand, since $\mathbb{Z}[M, D] \nsubseteq M\left(\mathbb{Z}^{n}\right)$ for a primitive digit set $D$, Theorems I and III are simple corollaries of (i).

In order to prove the Theorem, we need the following two lemmas.

Lemma 1. Let $M \in M_{n}(\mathbb{Z})$ be an expanding matrix, and let $D \subset \mathbb{Z}^{n}$ be a finite subset with $|D|=|\operatorname{det}(M)|$. If $\mu(T(M, D))>0$, then for each $l \in \mathbb{Z}^{n} \backslash\{0\}$, there exists a positive integer $k=k(l)$ such that $m_{M^{-k} D}(l)=0$, where

$$
m_{M^{-k} D}(t)=\frac{1}{|D|} \sum_{d \in D} e^{-2 \pi i\left\langle M^{-k} d, t\right\rangle} .
$$

This lemma is due to Lagarias and Wang [6, Theorem 2.1], who also proved the converse. For completeness, we provide a simple proof.

Proof of Lemma 1. Since $T(M, D)$ is an integral self-affine tile, it follows from (1.1) that $T=\bigcup_{d \in D}\left(M^{-1} T+M^{-1} d\right)$ is a measure-disjoint union, which 
can be written as

$$
\chi_{T}(t)=\sum_{d \in D} \chi_{T}(M t-d) .
$$

Taking the Fourier transform of (2.2), we have

$$
\widehat{\chi}_{T}(\xi)=m_{D}\left(M^{*-1} \xi\right) \widehat{\chi}_{T}\left(M^{*-1} \xi\right),
$$

which yields

$$
\widehat{\chi}_{T}(\xi)=m_{D}\left(M^{*-1} \xi\right) m_{D}\left(M^{*-2} \xi\right) \cdots m_{D}\left(M^{*-k} \xi\right) \widehat{\chi}_{T}\left(M^{*-k} \xi\right)
$$

by iteration, where $M^{*}$ denotes the conjugate transpose of $M$, in fact $M^{*}$ $=M^{T}$. Since $\lim _{k \rightarrow \infty} \widehat{\chi}_{T}\left(M^{*-k} \xi\right)=\widehat{\chi}_{T}(0)=\mu(T)$, it follows from (2.4) that

$$
\widehat{\chi}_{T}(\xi)=\mu(T) \prod_{j=1}^{\infty} m_{D}\left(M^{*-j} \xi\right)
$$

The infinite product in (2.5) converges absolutely for all $\xi \in \mathbb{R}^{n}$. For each $l \in \mathbb{Z}^{n} \backslash\{0\}$, taking $\xi=M^{* k} l$ in (2.4), we have

$$
\widehat{\chi}_{T}\left(M^{* k} l\right)=m_{D}\left(M^{*(k-1)} l\right) m_{D}\left(M^{*(k-2)} l\right) \cdots m_{D}(l) \widehat{\chi}_{T}(l)=\widehat{\chi}_{T}(l) .
$$

It follows from the Riemann-Lebesgue lemma and (2.6) that

$$
\widehat{\chi}_{T}(l)=\lim _{k \rightarrow \infty} \widehat{\chi}_{T}\left(M^{* k} l\right)=0,
$$

which gives the desired result by $(2.5)$.

For each integer $m>1$, let $\Gamma_{m}$ denote the lattice of integer relations among the $m$ th roots of unity, that is,

$$
\Gamma_{m}:=\left\{\left(a_{0}, a_{1}, \ldots, a_{m-1}\right) \in \mathbb{Z}^{m}: \sum_{k=0}^{m-1} a_{k} e^{2 \pi i k / m}=0\right\} .
$$

Let $m=p_{1}^{b_{1}} \cdots p_{r}^{b_{r}}$ be the standard decomposition into prime powers. For $\alpha_{1}=0,1, \ldots, m / p_{1}-1$, we define the point $P_{p_{1}, \alpha_{1}}=\left(u_{0}, u_{1}, \ldots, u_{m-1}\right)$ by

$$
\begin{aligned}
u_{\tau\left(m / p_{1}\right)+\alpha_{1}}=1, & & \tau=0,1, \ldots, p_{1}-1, \\
u_{\nu}=0 & & \text { if } \nu \neq \tau\left(m / p_{1}\right)+\alpha_{1} .
\end{aligned}
$$

Similarly, we define the points $P_{p_{2}, \alpha_{2}}\left(\alpha_{2}=0,1, \ldots, m / p_{2}-1\right), \ldots, P_{p_{r}, \alpha_{r}}$ $\left(\alpha_{r}=0,1, \ldots, m / p_{r}-1\right)$. Schoenberg [9] showed that every point $P=$ $\left(a_{0}, a_{1}, \ldots, a_{m-1}\right)$ of $\Gamma_{m}$ can be written as

$$
P=\sum_{\alpha_{1}=0}^{m / p_{1}-1} l_{p_{1}, \alpha_{1}} P_{p_{1}, \alpha_{1}}+\cdots+\sum_{\alpha_{r}=0}^{m / p_{r}-1} l_{p_{r}, \alpha_{r}} P_{p_{r}, \alpha_{r}},
$$

where $l_{p_{j}, \alpha_{j}}\left(\alpha_{j}=0,1, \ldots, m / p_{j}-1, j=1, \ldots, r\right)$ are all integers. 
In the special case when $m=p^{a}$ is a prime power, it follows from (2.8) and (2.9) that

$$
\begin{aligned}
& a_{0} \quad=a_{p^{a-1}} \quad=a_{2 p^{a-1}} \quad=\cdots=a_{(p-1) p^{a-1}} \quad=l_{p, 0} \\
& a_{1}=a_{p^{a-1}+1}=a_{2 p^{a-1}+1}=\cdots=a_{(p-1) p^{a-1}+1}=l_{p, 1}
\end{aligned}
$$

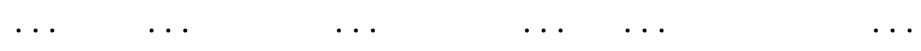

$$
\begin{aligned}
& a_{p^{a-1}-1}=a_{2 p^{a-1}-1}=a_{3 p^{a-1}-1}=\cdots=a_{p^{a}-1} \quad=l_{p, p^{a-1}-1} .
\end{aligned}
$$

Thus we have the following lemma.

Lemma 2. Let $m=p^{a}$ be a prime power. Then the coordinates of a point $P=\left(a_{0}, a_{1}, \ldots, a_{m-1}\right) \in \Gamma_{m}$ can be divided into $p^{a-1}$ groups of $p$ equal elements each.

Proof of Theorem. We introduce a lattice $\Lambda:=B^{-1}\left(\mathbb{Z}^{n}\right)$, which is $\widetilde{M}$ invariant. It can be proved that $D$ is a complete set of coset representatives of $\mathbb{Z}^{n} / M\left(\mathbb{Z}^{n}\right)$ if and only if $\widetilde{D}$ is a complete set of coset representatives of $\Lambda / \widetilde{M}(\Lambda)$. Here we use the same notation as above, and let $\widetilde{D}:=\left\{\widetilde{d}_{0}, \widetilde{d}_{1}, \ldots, \widetilde{d}_{p-1}\right\}$ with $\widetilde{d}_{0}=0$.

In view of Proposition 1 , we apply Lemma 1 to the pair $(\widetilde{M}, \widetilde{D})$, and conclude that for each $l \in \mathbb{Z}^{n} \backslash\{0\}$, there exists $k=k(l) \geq 1$ with $m_{\widetilde{M}^{-k} \widetilde{D}}(l)=0$. Let $\widetilde{M}^{\dagger}=p \widetilde{M}^{-1}$. Then $\widetilde{M}^{\dagger} \in M_{n}(\mathbb{Z})$ and

$$
\sum_{j=0}^{p-1} e^{2 \pi i\left\langle\left(\widetilde{M}^{\dagger}\right)^{k} \widetilde{d}_{j}, l\right\rangle / p^{k}}=0 .
$$

This is a sum of $p^{k}$ th roots of unity. In view of the fact that $\widetilde{d}_{0}=0$ and $p$ is a prime, it follows from Lemma 2 that

$$
\begin{aligned}
\left\{0,\left\langle\left(\widetilde{M}^{\dagger}\right)^{k} \widetilde{d}_{1}, l\right\rangle, \ldots,\right. & \left.\left\langle\left(\widetilde{M}^{\dagger}\right)^{k} \widetilde{d}_{p-1}, l\right\rangle\right\} \\
& \equiv\left\{0, p^{k-1}, 2 p^{k-1}, \ldots,(p-1) p^{k-1}\right\}\left(\bmod p^{k}\right) .
\end{aligned}
$$

CASE $1: \mathbb{Z}[M, D] \nsubseteq M\left(\mathbb{Z}^{n}\right)$. It is known that

$$
B\left(\mathbb{Z}^{n}\right) \subseteq M\left(\mathbb{Z}^{n}\right) \Leftrightarrow\left(M^{T}\right)^{-1}\left(\mathbb{Z}^{n}\right) \subseteq\left(B^{T}\right)^{-1}\left(\mathbb{Z}^{n}\right) .
$$

In this case, we choose $l^{\prime} \in \mathbb{Z}^{n} \backslash M^{T}\left(B^{T}\right)^{-1}\left(\mathbb{Z}^{n}\right)$. Then

$$
B^{T}\left(M^{\dagger}\right)^{T} l^{\prime} \notin p \mathbb{Z}^{n},
$$

where $M^{\dagger}=p M^{-1} \in M_{n}(\mathbb{Z})$.

Claim 1. There exists $w \in B\left(\mathbb{Z}^{n}\right)$ such that

$$
\left\langle M^{\dagger} w, l^{\prime}\right\rangle=w^{T}\left(M^{\dagger}\right)^{T} l^{\prime} \not \equiv 0(\bmod p) .
$$

Suppose that for all $w \in B\left(\mathbb{Z}^{n}\right)$ we have $w^{T}\left(M^{\dagger}\right)^{T} l^{\prime} \equiv 0(\bmod p)$. Let $e_{1}, \ldots, e_{n}$ be the standard basis of unit column vectors in $\mathbb{R}^{n}$. Taking $w=$ 
$B e_{1}, \ldots, B e_{n}$ respectively, we obtain

$$
e_{1}^{T} B^{T}\left(M^{\dagger}\right)^{T} l^{\prime}=p \xi_{1}, \quad \ldots, \quad e_{n}^{T} B^{T}\left(M^{\dagger}\right)^{T} l^{\prime}=p \xi_{n},
$$

where $\xi_{1}, \ldots, \xi_{n} \in \mathbb{Z}$. Hence $B^{T}\left(M^{\dagger}\right)^{T} l^{\prime}=p\left(\xi_{1}, \ldots, \xi_{n}\right)^{T} \in p \mathbb{Z}^{n}$ contradicts (2.13). Claim 1 is proved.

Since $\left(M^{\dagger}\right)^{T}=\left(B^{T}\right)^{-1}\left(\widetilde{M}^{\dagger}\right)^{T} B^{T}$, by letting

$$
\widetilde{w}:=B^{-1} w \in \mathbb{Z}^{n} \quad \text { and } \quad l:=B^{T} l^{\prime} \in B^{T}\left(\mathbb{Z}^{n}\right) \backslash \widetilde{M}^{T}\left(\mathbb{Z}^{n}\right),
$$

Claim 1 is equivalent to the following.

Claim $1^{\prime}$. There exists $\widetilde{w} \in \mathbb{Z}^{n}$ such that

$$
\left\langle\widetilde{M}^{\dagger} \widetilde{w}, l\right\rangle=\widetilde{w}^{T}\left(\widetilde{M}^{\dagger}\right)^{T} l \not \equiv 0(\bmod p) .
$$

Claim $1^{\prime}$ combined with $\mathbb{Z}^{n}=\mathbb{Z}[\widetilde{M}, \widetilde{D}]$ yields the following.

Claim 2. There exists $\widetilde{d}_{q} \in \widetilde{D}(1 \leq q \leq p-1)$ such that

$$
p \nmid\left\langle\widetilde{M}^{\dagger} \widetilde{d}_{q}, l\right\rangle .
$$

Furthermore, $\left\{0, \widetilde{d}_{q}, 2 \widetilde{d}_{q}, \ldots,(p-1) \widetilde{d}_{q}\right\}$ is a complete set of coset representatives of $\Lambda / \widetilde{M}(\Lambda)$.

In fact, we may write $\widetilde{w} \in \mathbb{Z}^{n}=\mathbb{Z}[\widetilde{M}, \widetilde{D}]$ as

$$
\widetilde{w}=\sum_{i=0}^{n-1} \sum_{j=0}^{p-1} n_{i, j} \widetilde{M}^{i} \widetilde{d}_{j} \quad\left(\widetilde{d}_{0}=0\right)
$$

for some integers $n_{i, j}(i=0,1, \ldots, n-1, j=0,1, \ldots, p-1)$. Substituting this in (2.16) and using $\widetilde{M} \widetilde{M}^{\dagger}=p I$, we have

$$
\sum_{j=0}^{p-1} n_{0, j}\left\langle\widetilde{M}^{\dagger} \widetilde{d}_{j}, l\right\rangle \not \equiv 0(\bmod p) .
$$

Thus there must exist $\widetilde{d}_{q} \in \widetilde{D}(1 \leq q \leq p-1)$ such that (2.17) holds.

For any integer $h$ with $|h|=1, \ldots, p-1,(2.17)$ gives

$$
p \nmid\left\langle h \widetilde{M}^{\dagger} \widetilde{d}_{q}, l\right\rangle,
$$

which shows that $h \widetilde{d}_{q} \notin \widetilde{M}(\Lambda)$, for if $h \widetilde{d}_{q}=\widetilde{M} \beta$ with $\beta \in \Lambda=B^{-1}\left(\mathbb{Z}^{n}\right)$, then $\left\langle h \widetilde{M}^{\dagger} \widetilde{d}_{q}, l\right\rangle=p\langle\beta, l\rangle \in p \mathbb{Z}$ contradicts (2.18). Hence Claim 2 holds.

It follows from Claim 2 that each $\lambda \in \Lambda$ has a unique representation

$$
\lambda=j_{0} \widetilde{d}_{q}+\widetilde{M} \varrho \quad \text { for some } 0 \leq j_{0} \leq p-1, \varrho \in \Lambda .
$$

Also from $\widetilde{M}^{\dagger} \widetilde{d}_{q} \in \mathbb{Z}^{n} \subseteq \Lambda$,

$$
\widetilde{M}^{\dagger} \widetilde{d}_{q}=j \widetilde{d}_{q}+\widetilde{M} \delta_{1} \quad \text { for some } 0 \leq j \leq p-1, \delta_{1} \in \Lambda \text {. }
$$


The assumption $p \mathbb{Z}^{n} \nsubseteq M^{2}\left(\mathbb{Z}^{n}\right)$ is equivalent to $\widetilde{M}^{\dagger}(\Lambda) \nsubseteq \widetilde{M}(\Lambda)$, which guarantees that $j \neq 0$ in $(2.20)$, for if $j=0$ in $(2.20)$, then $\widetilde{M}^{\dagger} \widetilde{d}_{q} \in \widetilde{M}(\Lambda)$, and (2.19) would give $\widetilde{M}^{\dagger} \lambda \in \widetilde{M}(\Lambda)$ for all $\lambda \in \Lambda$, a contradiction.

Now it follows from $(2.20)$ and $\widetilde{M}^{\dagger}(\Lambda) \subseteq \Lambda$ that for all $\sigma \geq 1$,

$$
(\widetilde{M} \dagger)^{\sigma} \widetilde{d}_{q}=j^{\sigma} \widetilde{d}_{q}+\widetilde{M} \delta_{\sigma} \quad \text { for } \delta_{\sigma} \in \Lambda,
$$

hence from (2.17),

$$
\begin{aligned}
\left\langle\left(\widetilde{M}^{\dagger}\right)^{\sigma} \widetilde{d}_{q}, l\right\rangle & =\left\langle\widetilde{M}^{\dagger}\left(j^{\sigma-1} \widetilde{d}_{q}+\widetilde{M} \delta_{\sigma-1}\right), l\right\rangle \\
& =j^{\sigma-1}\left\langle\widetilde{M}^{\dagger} \widetilde{d}_{q}, l\right\rangle+p\left\langle\delta_{\sigma-1}, l\right\rangle \not \equiv 0(\bmod p),
\end{aligned}
$$

where $\delta_{0}=0$ and $\left\langle\delta_{\sigma-1}, l\right\rangle \in \mathbb{Z}$ for $\delta_{\sigma-1} \in \Lambda$ and $l$ in (2.15).

Finally, comparing (2.12) and (2.22), we see that $k(l)=1$ for $l$ given in (2.15). Hence (2.12) becomes

$$
\left\{0,\left\langle\widetilde{M}^{\dagger} \widetilde{d}_{1}, l\right\rangle, \ldots,\left\langle\widetilde{M}^{\dagger} \widetilde{d}_{p-1}, l\right\rangle\right\} \equiv\{0,1, \ldots, p-1\}(\bmod p),
$$

for $l$ given in (2.15). In this case, if $\widetilde{d}_{i}-\widetilde{d}_{j}=\widetilde{M} \lambda$ for some $\lambda \in \Lambda$, then $\left\langle\widetilde{M}^{\dagger}\left(\widetilde{d}_{i}-\widetilde{d}_{j}\right), l\right\rangle=p\langle\lambda, l\rangle \in p \mathbb{Z}$ contradicts (2.23). Thus $\widetilde{D}$ is a complete set of coset representatives of $\Lambda / \widetilde{M}(\Lambda)$, and conclusion (i) of the Theorem holds.

CASE 2: $\mathbb{Z}[M, D] \subseteq M\left(\mathbb{Z}^{n}\right)$. From (1.3), we know that

$$
\mathbb{Z}[M, D]=B\left(\mathbb{Z}^{n}\right) \subseteq M\left(\mathbb{Z}^{n}\right) \Leftrightarrow D \subseteq M\left(\mathbb{Z}^{n}\right) .
$$

Let $D=M D^{\prime}$ and $\mathbb{Z}\left[M, D^{\prime}\right]=B^{\prime}\left(\mathbb{Z}^{n}\right)$. Then

$$
\mathbb{Z}[M, D]=M\left(B^{\prime}\left(\mathbb{Z}^{n}\right)\right) \text { and } T(M, D)=M\left(T\left(M, D^{\prime}\right)\right) .
$$

If $B^{\prime}\left(\mathbb{Z}^{n}\right) \nsubseteq M\left(\mathbb{Z}^{n}\right)$, the discussion in Case 1 applied to the pair $\left(M, D^{\prime}\right)$ shows that $D^{\prime}$ is a complete set of coset representatives of $\mathbb{Z}^{n} / M\left(\mathbb{Z}^{n}\right)$ and $D=M D^{\prime}$, i.e., conclusion (ii) of the Theorem holds with $\gamma=1$.

If $B^{\prime}\left(\mathbb{Z}^{n}\right) \subseteq M\left(\mathbb{Z}^{n}\right)$, then $\mathbb{Z}[M, D] \subseteq M^{2}\left(\mathbb{Z}^{n}\right)$. Let $D=M^{2} D^{\prime \prime}$ and $\mathbb{Z}\left[M, D^{\prime \prime}\right]=B^{\prime \prime}\left(\mathbb{Z}^{n}\right)$. Then

$$
\mathbb{Z}[M, D]=M^{2}\left(B^{\prime \prime}\left(\mathbb{Z}^{n}\right)\right) \text { and } T(M, D)=M^{2}\left(T\left(M, D^{\prime \prime}\right)\right) .
$$

The same argument applied to the case $B^{\prime \prime}\left(\mathbb{Z}^{n}\right) \nsubseteq M\left(\mathbb{Z}^{n}\right)$ gives conclusion (ii) with $\gamma=2$, while $B^{\prime \prime}\left(\mathbb{Z}^{n}\right) \subseteq M\left(\mathbb{Z}^{n}\right)$ needs the next step. After finitely many steps, we see that there exists an integer $\gamma \geq 1$ such that $\mathbb{Z}[M, D] \subseteq$ $M^{\gamma}\left(\mathbb{Z}^{n}\right)$ with $D:=M^{\gamma} D_{0}, \mathbb{Z}\left[M, D_{0}\right]:=B_{0}\left(\mathbb{Z}^{n}\right)$ but $B_{0}\left(\mathbb{Z}^{n}\right) \nsubseteq M\left(\mathbb{Z}^{n}\right)$. This is due to the fact that

$$
M^{\sigma}\left(\mathbb{Z}^{n}\right) \subseteq M^{\sigma-1}\left(\mathbb{Z}^{n}\right)(\sigma=1,2, \ldots) \text { and } \bigcap_{\sigma=1}^{\infty} M^{\sigma}\left(\mathbb{Z}^{n}\right)=\{0\} .
$$

See [10, Lemma 1]. Applying Case 1 to the pair $\left(M, D_{0}\right)$, we conclude that $D_{0}$ is a complete set of coset representatives of $\mathbb{Z}^{n} / M\left(\mathbb{Z}^{n}\right)$ and $D=M^{\gamma} D_{0}$. This completes the proof of the Theorem. 
3. Remarks and examples. (I) It should be pointed out that if $D$ is a complete set of coset representatives of $\mathbb{Z}^{n} / M\left(\mathbb{Z}^{n}\right)$, then $D$ is a standard digit set. However, the converse is not true (see [5, Remark 1]), even in the case when $|D|=|\operatorname{det}(M)|=p$ is a prime. Here we give a concrete example in higher dimensions. Note that in the one-dimensional case we have many such pairs $(M, D)$, for example

$$
M=[3] \quad \text { and } \quad D=\{0, l, 2 l\} \quad(l \in 3 \mathbb{Z} \backslash\{0\}) .
$$

However in higher dimensions, up to now each known digit set $D$ in an integral self-affine tile $T(M, D)$ is a complete set of coset representatives of $\mathbb{Z}^{n} / M\left(\mathbb{Z}^{n}\right)$, although the product-form digit set may give a different digit set when $|\operatorname{det}(M)|$ is not a prime (see [11]). Naturally one may ask whether there are other types of digit sets $D$ that also give integral self-affine tiles (see Remarks in [8, p. 1413]). The pair $(M, D)$ given below is the first such example in higher dimensions. From this example, one can see that the classification of all tile digit sets appears to be a difficult question even for prime determinants.

EXAMPLE. Let $p \geq 3$ be a prime. We consider the following expanding matrix $M \in M_{2}(\mathbb{Z})$ and digit set $D \subset \mathbb{Z}^{2}$ in $\mathbb{R}^{2}$ :

$$
M=\left[\begin{array}{ll}
0 & 1 \\
p & 0
\end{array}\right] \text { and } D=\left\{\left(\begin{array}{l}
0 \\
0
\end{array}\right),\left(\begin{array}{l}
1 \\
p
\end{array}\right), \ldots,\left(\begin{array}{c}
p-1 \\
p
\end{array}\right)\right\} .
$$

(i) Since

$$
\left(\begin{array}{l}
k_{1} \\
p
\end{array}\right)-\left(\begin{array}{l}
k_{2} \\
p
\end{array}\right)=M\left(\begin{array}{c}
0 \\
k_{1}-k_{2}
\end{array}\right)
$$

for any $1 \leq k_{1} \neq k_{2} \leq p-1$, we first note that $D$ is not a complete set of coset representatives of $\mathbb{Z}^{2} / M\left(\mathbb{Z}^{2}\right)$.

(ii) Observe that

$$
M(D)=\left\{\left(\begin{array}{l}
0 \\
0
\end{array}\right),\left(\begin{array}{c}
p \\
p
\end{array}\right),\left(\begin{array}{c}
p \\
2 p
\end{array}\right), \ldots,\left(\begin{array}{c}
p \\
(p-1) p
\end{array}\right)\right\} .
$$

Since

$$
\begin{gathered}
\left(\begin{array}{l}
2 \\
p
\end{array}\right)-\left(\begin{array}{l}
1 \\
p
\end{array}\right)=\left(\begin{array}{l}
1 \\
0
\end{array}\right), \\
\left(\begin{array}{l}
k \\
p
\end{array}\right)=\left(\begin{array}{l}
1 \\
p
\end{array}\right)+(k-1)\left(\begin{array}{l}
1 \\
0
\end{array}\right) \quad(k=3,4, \ldots, p-1), \\
\left(\begin{array}{c}
p \\
k_{1} p
\end{array}\right)=k_{1}\left(\begin{array}{l}
1 \\
p
\end{array}\right)+\left(p-k_{1}\right)\left(\begin{array}{l}
1 \\
0
\end{array}\right) \quad\left(k_{1}=1,2, \ldots, p-1\right),
\end{gathered}
$$


it follows from (1.3) that $\mathbb{Z}[M, D]=B\left(\mathbb{Z}^{2}\right)$ with

$$
B=\left[\begin{array}{ll}
1 & 2 \\
p & p
\end{array}\right] \quad \text { and } \quad B^{-1}=\left[\begin{array}{rr}
-1 & 2 / p \\
1 & -1 / p
\end{array}\right] .
$$

Hence

$$
\widetilde{M}=B^{-1} M B=\left[\begin{array}{ll}
2-p & 4-p \\
p-1 & p-2
\end{array}\right]
$$

and

$$
\widetilde{D}=B^{-1} D=\left\{\left(\begin{array}{l}
0 \\
0
\end{array}\right),\left(\begin{array}{l}
2-k \\
k-1
\end{array}\right): k=1, \ldots, p-1\right\} .
$$

(iii) Now

$$
\widetilde{M}^{-1}=\left[\begin{array}{ll}
(2-p) / p & (4-p) / p \\
(p-1) / p & (p-2) / p
\end{array}\right]
$$

and we have

$$
\begin{aligned}
& \widetilde{M}^{-1}\left(\begin{array}{l}
2-k \\
k-1
\end{array}\right)=\left(\begin{array}{c}
(2 k-p) / p \\
(p-k) / p
\end{array}\right) \notin \mathbb{Z}^{2} \quad(k=1, \ldots, p-1), \\
& \widetilde{M}^{-1}\left\{\left(\begin{array}{c}
2-k_{1} \\
k_{1}-1
\end{array}\right)-\left(\begin{array}{c}
2-k_{2} \\
k_{2}-1
\end{array}\right)\right\}=\left(\begin{array}{c}
2\left(k_{1}-k_{2}\right) / p \\
\left(k_{2}-k_{1}\right) / p
\end{array}\right) \notin \mathbb{Z}^{2},
\end{aligned}
$$

where $1 \leq k_{1} \neq k_{2} \leq p-1$. Thus $\widetilde{D}$ is a complete set of coset representatives of $\mathbb{Z}^{2} / \widetilde{M}\left(\mathbb{Z}^{2}\right)$. Furthermore, $\mu(T(\widetilde{M}, \widetilde{D}))>0$, which gives $\mu(T(M, D))=$ $|\operatorname{det}(B)| \mu(T(\widetilde{M}, \widetilde{D}))>0$.

(iv) The fact that $\mu(T(M, D))=p \mu(T(\widetilde{M}, \widetilde{D}))=p$ can be proved by applying Proposition 2 below, essentially due to Lagarias and Wang. Therefore we have a pair $(M, D)$ such that the following conditions hold:

(1) $|\operatorname{det}(M)|=p$ is a prime and $p \mathbb{Z}^{n}=M^{2}\left(\mathbb{Z}^{n}\right)$.

(2) $\mu(T(M, D))=p$.

(3) $D$ is a standard digit set.

(4) $D$ is not a complete set of coset representatives of $\mathbb{Z}^{n} / M\left(\mathbb{Z}^{n}\right)$.

(II) It is known that a Haar-type orthonormal wavelet basis can be constructed from an integral self-affine tile $T(\widetilde{M}, \widetilde{D})$ with $\mu(T(\widetilde{M}, \widetilde{D}))=1$ (see [12]). There are several conditions to guarantee $\mu(T(\widetilde{M}, \widetilde{D}))=1$. We state the following proposition which can be applied to prove $\mu(T(M, D))=$ $|\operatorname{det}(B)| \mu(T(\widetilde{M}, \widetilde{D}))=|\operatorname{det}(B)|$ easily in the case of prime determinant.

Proposition 2. Let $\widetilde{M} \in M_{n}(\mathbb{Z})$ be an expanding matrix and $\widetilde{D} \subset \mathbb{Z}^{n}$ with $|\widetilde{D}|=|\operatorname{det}(\widetilde{M})|=p$. If $p$ is a prime, then $\mu(T(\widetilde{M}, \widetilde{D}))=1$ if and only 
if $\widetilde{D}$ is primitive and is a complete set of coset representatives of $\mathbb{Z}^{n} / \widetilde{M}\left(\mathbb{Z}^{n}\right)$. Furthermore, in the case when $p=2$, a necessary and sufficient condition for $\mu(T(\widetilde{M}, \widetilde{D}))=1$ is $\mathbb{Z}[\widetilde{M}, \widetilde{D}]=\mathbb{Z}^{n}$.

Proof. Since $|\operatorname{det}(\widetilde{M})|=p$ is a prime, the characteristic polynomial of $\widetilde{M}$ is irreducible over $\mathbb{Q}$, and the first assertion follows from Theorems 3.3 and 5.3 in [11]. The second conclusion follows from the result that if $\widetilde{M} \in$ $M_{n}(\mathbb{Z})$ is a (not necessarily expanding) matrix with $|\operatorname{det}(\widetilde{M})|=2$, then each primitive digit set for $\widetilde{M}$ is a complete set of coset representatives of $\mathbb{Z}^{n} / \widetilde{M}\left(\mathbb{Z}^{n}\right)$ (see [7, p. 191]). In fact, without loss of generality, we let $\widetilde{D}=\{0, d\}$ be a primitive digit set for $\widetilde{M}$. If $\widetilde{D}$ is not a complete set of coset representatives of $\mathbb{Z}^{n} / \widetilde{M}\left(\mathbb{Z}^{n}\right)$, then $d=\widetilde{M} l$ for some $l \in \mathbb{Z}^{n}$. From (1.3),

$$
\mathbb{Z}^{n}=\mathbb{Z}\left[\widetilde{M} l, \widetilde{M}^{2} l, \ldots, \widetilde{M^{n}} l\right] \subseteq \widetilde{M}\left(\mathbb{Z}^{n}\right) \subset \mathbb{Z}^{n},
$$

which contradicts the fact that $|\operatorname{det}(\widetilde{M})|=2$. This completes the proof.

Note that it follows from Propositions 1 and 2 that $T(M, D)$ being an integral self-affine tile, i.e. the condition $\mu(T(M, D))>0$ in Theorem II essentially gives $\mu(T(M, D))=|\operatorname{det}(B)|$, while the same condition in Theorems I and III (the case of primitive tile) essentially gives $\mu(T(M, D))=1$. In the case when $p=2$, the conclusions of Theorems II and III hold without the condition $p \mathbb{Z}^{n} \nsubseteq M^{2}\left(\mathbb{Z}^{n}\right)$. On the other hand, the above Example is also suitable for $p=2$. The case $|\operatorname{det}(M)|=2$ gives $D=\{0, d\}$ with only one digit $d$ to choose. For a given expanding matrix $M \in M_{n}(\mathbb{Z})$ with $|\operatorname{det}(M)|=2$, the digit set $D=\{0, M l\}$ is neither a primitive set nor a complete set of coset representatives of $\mathbb{Z}^{n} / M\left(\mathbb{Z}^{n}\right)$, for any $l \in \mathbb{Z}^{n} \backslash\{0\}$. However $\mu(T(M, D))>1$ and $D$ is a standard digit set. To see this, note that

$$
\begin{aligned}
& \mathbb{Z}[M, D]=B\left(\mathbb{Z}^{n}\right)=\mathbb{Z}\left[M l, M^{2} l, \ldots, M^{n} l\right] \subseteq M\left(\mathbb{Z}^{n}\right) \subset \mathbb{Z}^{n}, \\
& \widetilde{M}=B^{-1} M B, \quad \widetilde{D}=B^{-1} D=\left\{0, B^{-1} M l\right\}, \\
& \mathbb{Z}[\widetilde{M}, \widetilde{D}]=\mathbb{Z}\left[B^{-1} M l, B^{-1} M^{2} l, \ldots, B^{-1} M^{n} l\right]=B^{-1}(\mathbb{Z}[M, D]) .
\end{aligned}
$$

Hence $\mathbb{Z}[\widetilde{M}, \widetilde{D}]=\mathbb{Z}^{n}, \widetilde{D}$ is a complete set of coset representatives of $\mathbb{Z}^{n} / \widetilde{M}\left(\mathbb{Z}^{n}\right)$ and

$$
\mu(T(M, D))=|\operatorname{det}(B)| \mu(T(\widetilde{M}, \widetilde{D}))=|\operatorname{det}(B)|>1 .
$$

Therefore we also have many such pairs $(M, D)$ satisfying the desired conditions in higher dimensions, for example

$$
M=\left[\begin{array}{rrrr}
0 & 1 & 0 & 0 \\
0 & 0 & 1 & 0 \\
0 & 0 & -1 & 2 \\
-1 & 0 & -1 & 1
\end{array}\right] \text { and } D=\left\{\left(\begin{array}{l}
0 \\
0 \\
0 \\
0
\end{array}\right),\left(\begin{array}{l}
1 \\
0 \\
0 \\
0
\end{array}\right)\right\} .
$$


In (3.8), $M$ is an expanding matrix with $\operatorname{det}(M)=2, D=\{0, M l\}$ with $l=(0,1,0,0)^{T}$, and $B$ and $\widetilde{M}$ are given by

$$
B=\left[\begin{array}{rrrr}
1 & 0 & 0 & 0 \\
0 & -2 & 0 & 0 \\
0 & 0 & -2 & 0 \\
0 & 1 & -1 & -1
\end{array}\right] \text { and } \widetilde{M}=\left[\begin{array}{rrrr}
0 & -2 & 0 & 0 \\
0 & 0 & 1 & 0 \\
0 & -1 & 0 & 1 \\
1 & 0 & 0 & 0
\end{array}\right]
$$

So $\widetilde{D}=B^{-1} D=D$. Now, $D$ is not a complete set of coset representatives of $\mathbb{Z}^{4} / M\left(\mathbb{Z}^{4}\right)$, but $\widetilde{D}=D$ is a complete set of coset representatives of $\mathbb{Z}^{4} / \widetilde{M}\left(\mathbb{Z}^{4}\right)$ and $\mu(T(M, D))=4 \mu(T(\widetilde{M}, \widetilde{D}))=4$.

(III) For the matrix $M$ in (3.8), there is no digit set $D \subset \mathbb{Z}^{4}$ such that the pair $(M, D)$ gives a Haar-type wavelet basis of $\mathbb{R}^{4}$; understanding the reasons for this fact requires some knowledge of algebraic number theory (see [7]). We observe from Proposition 2 that this is equivalent to saying that there is no digit set $D \subset \mathbb{Z}^{4}$ such that $\mathbb{Z}[M, D]=\mathbb{Z}^{4}$. In fact, for the matrix $M$ in (3.8) and any digit set $D=\left\{(0,0,0,0)^{T},(\alpha, \beta, \gamma, \eta)^{T}\right\} \subset \mathbb{Z}^{4}$, we have $\mathbb{Z}[M, D]=B\left(\mathbb{Z}^{4}\right)$ with

$$
B=\left[\begin{array}{cccc}
\alpha & \beta & \gamma & 2 \eta-\gamma \\
\beta & \gamma & 2 \eta-\gamma & -2 \alpha-\gamma \\
\gamma & 2 \eta-\gamma & -2 \alpha-\gamma & -2 \beta-2 \eta+\gamma \\
\eta & \eta-\alpha-\gamma & -\alpha-\beta-\eta & \alpha-\beta-\eta
\end{array}\right] .
$$

A computation shows that $\operatorname{det}(B)$ is an even integer. So $\mathbb{Z}[M, D]=\mathbb{Z}^{4}$ cannot hold. This shows that for a given expanding matrix $M \in M_{n}(\mathbb{Z})$, it is not always possible to find a digit set $D$ such that $D$ is primitive with respect to $M$.

(IV) Finally, we point out that the first example given by

$$
M=\left[\begin{array}{rr}
0 & 1 \\
-3 & 2
\end{array}\right] \text { and } D=\left\{\left(\begin{array}{l}
0 \\
0
\end{array}\right),\left(\begin{array}{l}
1 \\
0
\end{array}\right),\left(\begin{array}{r}
-1 \\
5
\end{array}\right)\right\}
$$

in $[2$, Figure 1] cannot produce an integral self-affine tile $T(M, D)$, because $\mu(T(M, D))=0$. In fact, for the pair $(M, D)$ in $(3.11)$, we have

$$
M(D)=\left\{\left(\begin{array}{l}
0 \\
0
\end{array}\right),\left(\begin{array}{c}
0 \\
-3
\end{array}\right),\left(\begin{array}{c}
5 \\
13
\end{array}\right)\right\}
$$

and for any $k_{1}, k_{2} \in \mathbb{Z}$,

$$
\left(k_{1}-k_{2}\right)\left(\begin{array}{l}
1 \\
0
\end{array}\right)-2 k_{2}\left(\begin{array}{r}
0 \\
-3
\end{array}\right)-k_{2}\left(\begin{array}{r}
-1 \\
5
\end{array}\right)=\left(\begin{array}{l}
k_{1} \\
k_{2}
\end{array}\right),
$$


which shows that $\mathbb{Z}[M, D]=\mathbb{Z}^{2}$. On the other hand, one can check that $|\operatorname{det}(M)|=p=3$ and $p \mathbb{Z}^{2} \nsubseteq M^{2}\left(\mathbb{Z}^{2}\right)$. If $\mu(T(M, D))>0$, then statement (i) of the Theorem shows that $D$ is a complete set of coset representatives of $\mathbb{Z}^{2} / M\left(\mathbb{Z}^{2}\right)$. But

$$
\left(\begin{array}{l}
1 \\
0
\end{array}\right)-\left(\begin{array}{r}
-1 \\
5
\end{array}\right)=M\left(\begin{array}{l}
3 \\
2
\end{array}\right)
$$

which proves that $\mu(T(M, D))=0$, and $T(M, D)$ with $(M, D)$ given by (3.11) is not a tile.

Acknowledgements. I am indebted to Professor K. S. Lau for his valuable suggestions. I also thank Professor Y. Wang for a helpful discussion on the subject during his visit at CUHK.

\section{References}

[1] C. Bandt, Self-similar sets 5. Integer matrices and fractal tilings of $\mathbb{R}^{n}$, Proc. Amer. Math. Soc. 112 (1991), 549-562.

[2] D. Hacon, N. C. Saldanhan and J. J. P. Veerman, Remarks on self-affine tilings, Experiment. Math. 3 (1994), 317-327.

[3] J. E. Hutchinson, Fractals and self-similarity, Indiana Univ. Math. J. 30 (1981), $713-747$.

[4] R. Kenyon, Self-replicating tilings, in: Symbolic Dynamics and Its Applications, P. Walters (ed.), Contemp. Math. 135, Amer. Math. Soc., 1992, 239-263.

[5] I. Kirat and K. S. Lau, Classification of integral expanding matrices and self-affine tiles, Discrete Comput. Geom. 28 (2002), 49-73.

[6] J. C. Lagarias and Y. Wang, Integral self-affine tiles in $\mathbb{R}^{n}$. I: Standard and nonstandard digit sets, J. London Math. Soc. 54 (1996), 161-179.

[7] - - - Haar basis for $L^{2}\left(\mathbb{R}^{n}\right)$ and algebraic number theory, J. Number Theory 57 (1996), 181-197; Corrigendum/Addendum, 76 (1999), 330-336.

[8] K. S. Lau and H. Rao, On one-dimensional self-similar tilings and pq-tiles, Trans. Amer. Math. Soc. 355 (2003), 1401-1414.

[9] I. J. Schoenberg, A note on the cyclotomic polynomial, Mathematika 11 (1964), $131-136$.

[10] A. Vince, Replicating tessellations, SIAM J. Discrete Math. 6 (1993), 501-521.

[11] Y. Wang, Self-affine tiles, in: Advances in Wavelets, K. S. Lau (ed.), Springer, Singapore, 1999, 261-282.

[12] P. Wojtaszczyk, A Mathematical Introduction to Wavelets, Cambridge Univ. Press, Cambridge, 1997.

College of Mathematics and Information Science

Shaanxi Normal University

Xi'an 710062, P.R. China

E-mail: jllimath@yahoo.com.cn

Received December 9, 2005

Revised version September 26, 2006 